\title{
Copyright Protection for Digital Colour Images Using Dual Watermarking Technique by Applying Improved DWT, DCT and SVD
}

\author{
Lakshmi Priya A., *, S. Letitia ${ }^{2}$ \\ ${ }^{1}$ Department of ECE, Global Institute of Engineering and Technology, Vellore, Tamil Nadu, India \\ ${ }^{2}$ Department of ECE, ThanthaiPeriyar Government Institute of Engineering and Technology, Vellore, Tamil Nadu, India \\ Email address: \\ a.v.lakshmipriyaa@gmail.com (Lakshmi P. A.), letitiadurai@gmail.com (S. Letitia) \\ ${ }^{*}$ Corresponding author
}

\section{To cite this article:}

Lakshmi Priya A., S. Letitia. Copyright Protection for Digital Colour Images Using Dual Watermarking Technique by Applying Improved DWT, DCT and SVD. Journal of Electrical and Electronic Engineering. Vol. 4, No. 5, 2016, pp. 120-130. doi: 10.11648/j.jeee.20160405.16

Received: December 8, 2015; Accepted: December 17, 2015; Published: November 19, 2016

\begin{abstract}
The main objective of the majority of research in Digital Image Watermarking technique is to improve the robustness of attacks.In this paper, a new algorithm has been proposed based on Discrete Wavelet Transform, Discrete Cosine Transformation in association with Singular Value Decomposition for embedding the dual watermark which provides an efficient copyright protection. Here the primary watermark is embedded into the secondary watermark and the resultant image is used as a watermark for the host or cover image. The primary and secondary watermarks are retrieved back by using an efficient watermark retrieving algorithm. During the processes of embedding the scaling factor is varied to improve the quality of watermarked image. In this proposed algorithm the watermarked image is extracted back with same quality, high peak signal to noise ratio and normalized correlation coefficients. The quality of the extracted watermarks is authenticated using Peak Signal to Noise Ratio and Normalized Correlation Coefficient. Compared with previous methods the proposed algorithm has a significantly higher robustness against various geometrical and removal attacks.Nearly 28 attacks like Cropping, Rotating at various angles, Sharpening, Motion Blur, rescaling, resizing and adding noise like Salt and Pepper noise, Gaussian noise, Spackle noise, filters like average filter, median filters are attempted. Even after attempted all such attacks both the watermarks are retrieved back with the same quality and high normalized correlation coefficient.
\end{abstract}

Keywords: Discrete Wavelet Transform (DWT), Discrete Cosine Transform (DCT), Singular Value Decomposition (SVD), Peak Signal to Noise Ratio (PSNR), Normalized Correlation (NC)

\section{Introduction}

In many applications like military image database, confidential video conferences, personal online photographs and medical imaging are stored as well as transmitted through internet and multimedia. The recent proliferations are copying data illegally, tampering and copyright protection in internet and multimedia. To protect the copyrights of such digital multimedia contents a standard elucidation is required.Digital image watermarking is one of the emerged solutions for protecting such multimedia contents [1].An effective Image watermarking has to meet the basic requirements of robustness, embedding capacity, visual imperceptibility and uniqueness for copyright protection, content archiving, meta-data insertion, broadcast monitoring, tamper detection and digital fingerprinting [2, 3]. Generally the image watermarking techniques are classified as spatial domain watermarking techniques and frequency domain watermarking techniques.Spatial domain techniques are economical and easy to implement where the watermarks are directly inserted by simply varying the pixel values of the cover image. Hence it can be easily affected by an assortment of attacks. Where as in frequency domain techniques, the spatial domain information is converted into frequency domain coefficients by using the transformation 
techniques like Discrete Fourier Transform (DFT), Discrete Cosine Transform (DCT), Discrete Wavelet Transform (DWT) and Singular Value Decomposition (SVD). These methods provide higher image imperceptibility and robustness [4, 5].

Robustness are further divided into two categories: Robust and fragile watermarking. Robust watermarking is used to measure the ability of the watermark to resist common image processing operations like cropping, scaling, filtering, additive noise and JPEG compression too provides ownership claims. Whereas fragile watermarking is used to authenticate multimedia content which is to identify and report all feasible tampering in the watermarked digital media [6]. Many research works show that combining two are more transformation techniques to improve the quality of the watermark. The concept this is while combining various techniques the drawbacks of one is compensated by other. Sticking to the idea, the transformation techniques DWT, DCT and SVD are combined to develop an efficient embedding algorithm.

The concept of dual watermarking increases the security of the watermark further yet it provides a reliable solution to two foremost security concerns: information recovery and ownership identification.Any granular user defined crucial information may be used as a primary watermark. In order to resolve ownership authentication any secured attribute like fingerprint or secret keys only known to the owner can be used as secondary watermark. The primary watermark is embedded into the secondary watermark and the resultant image is used as a watermark for the host or cover image by using improved DWT, DCT and SVD embedding algorithm. The primary and secondary watermarks are retrieved back by using an efficient watermark retrieving algorithm.

\section{Preliminaries}

\subsection{Discrete Wavelet Transform (DWT)}

Due to the good energy compaction property of wavelet transform, it becomes an important tool in image processing and watermarking. Many research works in this field brought out numerous algorithms to produce efficient wavelets. The DWT separates an image into four parts, lower resolution approximation component (LL) is acquired by low pass filtering both rows and columns and contains a rough portrayal of an image, diagonal (HH) component contains a high frequency component along the diagonals. Horizontal (HL) and Vertical (LH) is mid frequencies obtained by low pass filtering in one direction and high pass filtering in another direction. The large amount of energy is concentrated in LL sub bands and so embedding in the LL sub band increases the robustness of the watermark significantly. Hence, in this algorithm LL is chosen for embedding process $[8,9]$.

\subsection{Discrete Cosine Transform (DCT)}

DCT is one of the most fascinating linear transformation techniques in signal and image processing. The DCT embeds the watermark in perceptually significant segment of the image is more advantageous because the compression algorithm only removes the insignificant segment of the image. DCT is mainly preferred because it has semantically meaningful watermark pattern, good perceptual invisibility, adequate robustness, reasonable complexity and execution time. The 2D discrete cosine transform and its inverse transforms are mathematically represented in equation 1 and 2 .

$$
\begin{aligned}
& F(k, l)=\frac{2}{\sqrt{N}} \alpha(k) \alpha(l) \sum_{m=0}^{N-1} \sum_{n=0}^{N-1} f(m, n) \cos \left\{\frac{(2 m+1) k \pi}{2 N}\right\} \cos \left\{\frac{(2 n+1) l \pi}{2 N}\right\} \\
& f(m, n)=\frac{2}{\sqrt{N}} \sum_{k=0}^{N-1} \sum_{l=0}^{N-1} \alpha(k) \alpha(l) F(k, l) \cos \left\{\frac{(2 m+1) k \pi}{2 N}\right\} \cos \left\{\frac{(2 n+1) l \pi}{2 N}\right\}
\end{aligned}
$$

Where $f(m, n)$ is the pixel representation in spatial domain and $F(k, l)$ is its DCT coefficient, $m$ and $n$ represents the block size $[10,11]$.

$$
\alpha(k) \alpha(l)=\left\{\begin{array}{c}
\frac{1}{\sqrt{2}} \text { if } u, v=0 \\
1 \text { else }
\end{array}\right.
$$

\subsection{Singular Value Decomposition (SVD)}

SVD is a linear algebraic transformation technique which is used in watermarking; it modifies the singular values of the entire host image by embedding the watermark data. The singular value specifies the luminance value of an image and the singular vector specifies the geometry of the image layer. The robustness of an image is increased by applying SVD because due to attacks, there is a large change in image which results in a very small change in watermarks.Let $\mathrm{S}$ denotes the real matrix of order $m \times n$. The SVD of $\mathrm{S}$ in denoted as, $S=u \sigma v^{T}$ where $\sigma$ is the diagonal matrix, $u$ and $v$ is orthogonal matrixes. SVD has fascinating properties:the transformation applied to an image may have an arbitrary size, which is not restricted to square image. And the singular value of image posses the intrinsic algebraic property of the image [12-14].

\section{Proposed Algorithm}

The watermark embedding in this proposed algorithm comprises of two stages. In the first stage the host image is divided into $\mathrm{R}, \mathrm{G}$ and Bplanes. In which the Blue plane image is chosen for analysis because any change happened in the blue plane is not much sensible to Human vision $[16,17]$. DWT and DCT are combined with SVD to improve the quality and robustness of the watermark. Many research works proved that the quality of the watermark is improved by combining more than one transformation techniques because, while various techniques are combined the 
drawbacks of the one is met by another [20-23]. The host image is subjected into three levels of haar wavelet transformation technique. This decomposes the host image into four sub bands of identical bandwidth namely LL, LH, HL and HH. The magnitude of LL's coefficients is very high at each level of decomposition and most of the energy is concentrated in LL sub bands. Thus, embedding in LL sub band increases the robustness of the watermark significantly. The reason for DWT preferenceis it has an important property called spatial locality. While embedding the watermark the wavelet transformation provides both frequency and spatial information of an image. Next DCT is applied to all sub-band of DWT because it has good perceptual invisibility and robustness. To increase the robustness of the watermark,SVD is applied to all its subbands at last.

Similarly two levels of haar wavelet transformation are applied to both watermarks. Any user specified information is chosen as primary watermark. In order to resolve ownership authentication fingerprint is used as secondary watermark. The DCT and SVD are applied further to both watermark images. The primary watermark is embedded into the secondary watermark and the resultant watermarked image is used as a watermark for the host image. Inverse SVD, Inverse DCT and Inverse DWT areperformed to obtain the watermarked image.

In the second stage the scaling factor is slightly varied, which depends on the affected watermarked image, to improve the normalised correlation coefficientby using the same algorithm discussed earlier. On choosing a proper scaling factor, the robustness with minimum loss in quality can be improved because single scaling factor may not be suitable for all type of images when it is brutally corrupted by attacks. Hence a new approach is attempted to increase the quality and correlation coefficient of the retrieved watermark by varying the scaling factor of the watermarked image during extraction.

\section{Embedding Algorithm}

1. The image is subjected to 3 levelsDWT and LL subband is chosen.

2. Similarly 2 levels of DWT are applied to primary and secondary watermarks.

3. DCT is applied all sub bands of Host image as well as the Watermark images,

4. SVD is used to partition these values into $u, v$ and $\sigma$.

$$
S^{i}=u_{i} S_{i} v_{i}^{T}
$$

where $i=h, w 1, w 2$, h represents the singular values of host image $w 1$ and $w 2$ are the singular values of primary and secondary watermark.

5. Modify the singular values of the diagonal matrix as,

$$
\begin{gathered}
s_{i}^{\prime}=s_{w 1}+s_{w 2} \\
s_{i}^{\prime \prime}=s_{1}+\alpha s_{i}^{\prime} \stackrel{\text { Inverse }}{\Longleftrightarrow} u_{k}^{\prime} s_{k}^{\prime} v_{k}^{\prime T}
\end{gathered}
$$

$\mathrm{S}_{\mathrm{i}}$ " represents the new singular value obtained from host and watermarks.

6. Then apply inverse DCT and inverse DWT to obtain the watermarked image.

Fig. 1.representsthe Embedding process where the Host image and Watermarks are embedded.

\section{Extraction Algorithm}

1. The watermarked image $\mathrm{Wk}$ is decomposed by applying 3 level DWT.

2. Apply DCT and SVD to all sub-bands.

3. Extract the singular value matrix S"wk of the watermarked image $\mathrm{Wk}$.

$$
\mathrm{S}{ }^{\prime} w \mathrm{wk}=\left(\mathrm{S}^{\prime} w \mathrm{w}-\mathrm{Swk}{ }^{*}\right) / \alpha
$$

$\mathrm{S}_{\mathrm{wk}}{ }_{\mathrm{w}}-$ Diagonal matrix of Watermarked image

$\mathrm{S}_{\text {wk }}$ - Diagonal matrix of Extracted Watermark

$\alpha$ - Scaling factor

4. From $S$ "wk segregate the singular values of primary and secondary watermarks.

5. Compute inverse SVD, DCT and DWT to obtain watermark images back.

Fig 2 represents the extraction algorithm which retrieves the watermark images back after attempting with all feasible attacks.

\section{Experimental Result}

The proposed Discrete Wavelet Transform, Discrete Cosine Transform combined with a Singular Value Decomposition algorithm was examined with constant $\alpha$ and varying $\alpha$. The Host is divided into Red, Blue and Green components. The Blue component alone is chosen for analysis. The Blue components so chosen because the characters utilized are not sensible to Human vision system [17]. The watermarking is done as per the embedding algorithm discussed in section 4. The robustness of the watermarked images are examined with all possible attacks, nearly 28 attacks is attempted to prove the resistance of primary as well as secondary watermarks. The proposed algorithm proves that in all the above attacks the watermark is retrieved back with better quality. The Peak Signal to Noise Ratio (PSNR) is used to measure the distortion between host image and watermarked image. The Normalized Correlation (NC) is used to find the similarities between the host image and watermarked image. The Peak Signal to Noise Ratio (PSNR), Mean Square Error (MSE) and Normalized correlation (NC) have been calculated by using,

$$
\mathrm{MSE}=\frac{1}{m n} \sum_{i=1}^{m} \sum_{j=1}^{n}\left(x(i, j)-\mathrm{W}_{\mathrm{K}}^{*}(\mathrm{i}, \mathrm{j})^{2}\right.
$$

The PSNR is found using,

$$
\mathrm{PSNR}=10 \log 10\left(\frac{255^{2}}{M S E}\right)
$$


and $\mathrm{NC}$ is given as,

$$
\mathrm{NC}=\frac{\sum_{i}^{m} \sum_{j}^{n}\left[x(i, j) w_{k}^{*}(i, j)\right]}{\sqrt{\sum_{i}^{m} \sum_{j}^{n}(x(i, j))^{2}} \sqrt{\sum_{i}^{m} \sum_{j}^{n}\left(w_{k}^{*}(i, j)\right)^{2}}}
$$

where $x(i, j)$ and $W_{K}^{*}(i, j)$ are the pixel values of host image and watermarked image respectively and $m, n$ are used to specify the row and column of the images respectively. The algorithm is attempted with number of Host images and watermark images and at last the below given images are chosen.

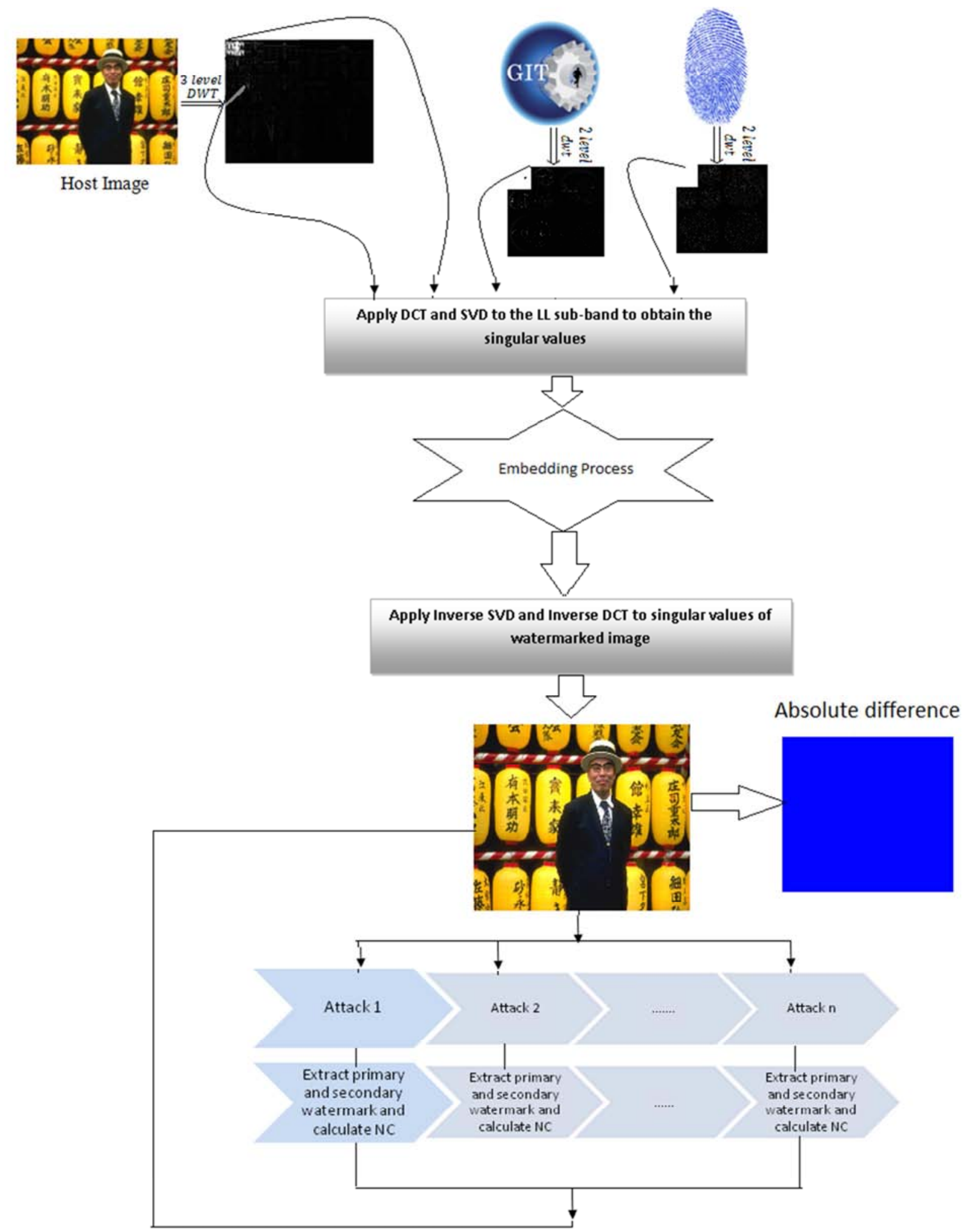

Fig. 1. Embedding technique. 


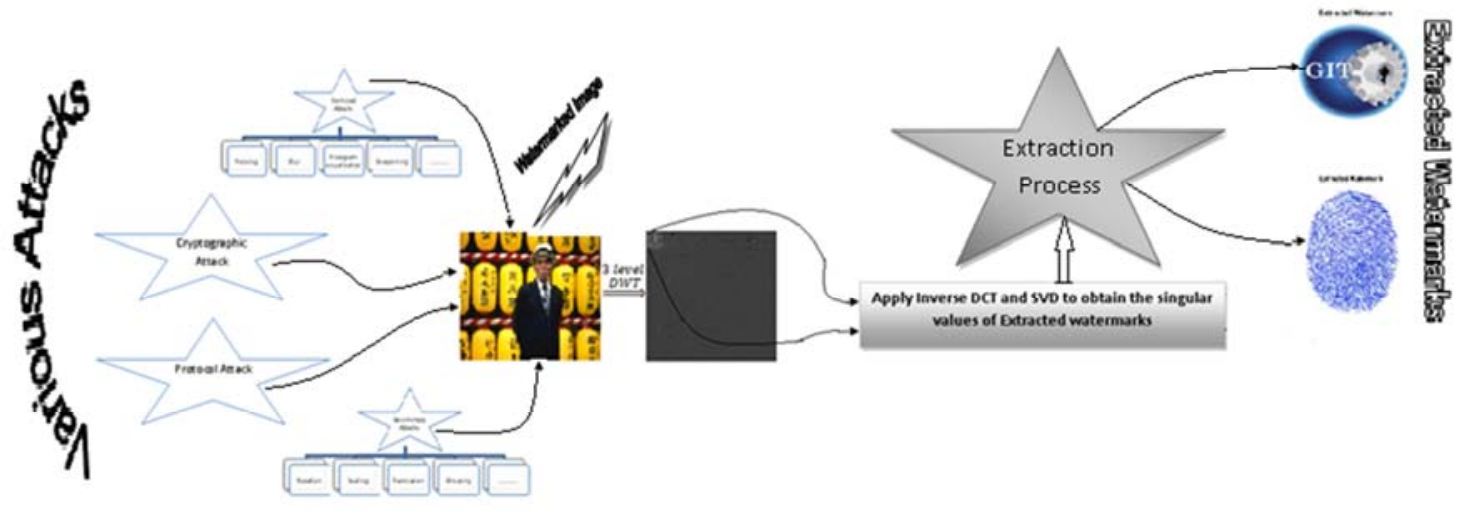

Fig. 2. Process of extraction.

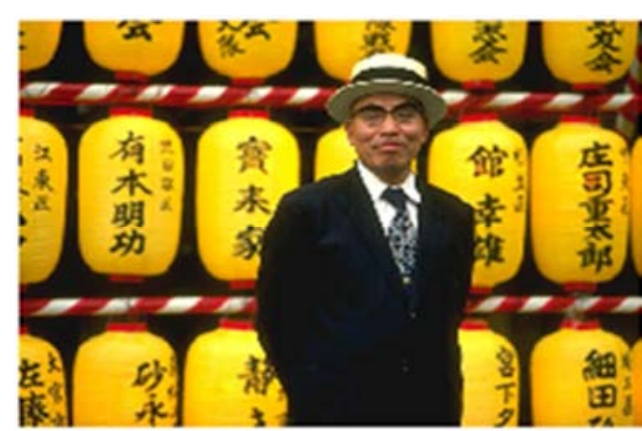

Fig. 3. Host Image.

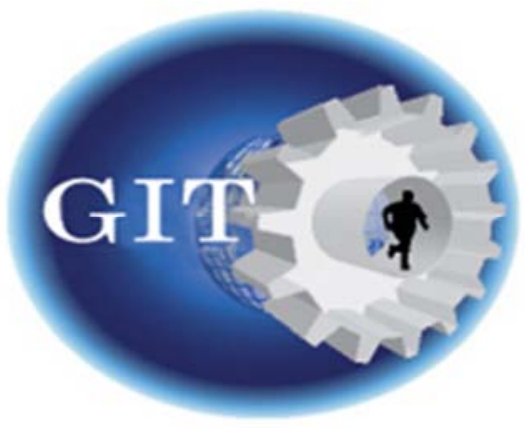

Fig. 4. Primary Watermark.

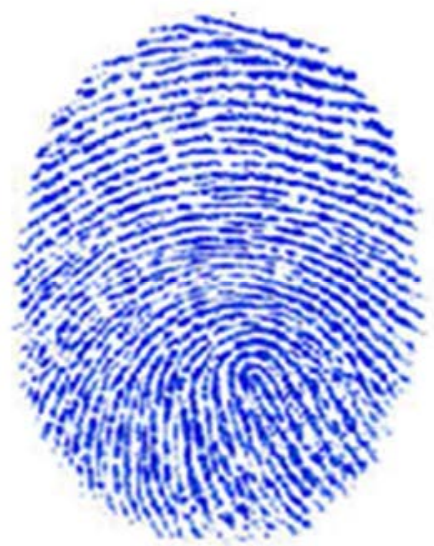

Fig. 5. Secondary Watermark.
The Primary watermark is embedded with secondary watermark the resultant image is used as watermark for the host. The quality of the watermarked is best maintained with PSNR $=44.199$ and $\mathrm{NC}=0.9998$. Both embedded watermarks are extracted back with best PSNR and NC as shown in Table.1. This shows that good imperceptibility is obtained by the proposed technique compared with the existing techniques $[24,25]$.

Table 1. PSNR and NC values of Watermarked and extracted watermarks.

Watermarked
Image

Thus the watermark existence is verified. The absolute difference shows that there is no difference between the original and extracted watermarks and the value of $\mathrm{NC}=1$ proves the quality.

The robustness and resistance of the proposed technique is also verified with variety of attacks like Cropping with various dimensions, Rotation at various angles, Sharpening, Motion blur, Warping and added noises like Gaussian with varying variance, Poisson noise, Salt and Pepper noise, Speckle noise and various filtering like Median and Averaging, Histogram equalization, Rescaling and Contrast adjustment.Almost 28 attacks have been attempted to authenticate the resistance of watermarked image. 
Table 2. Affected watermarked \&Extracted watermarks after applying various attacks.

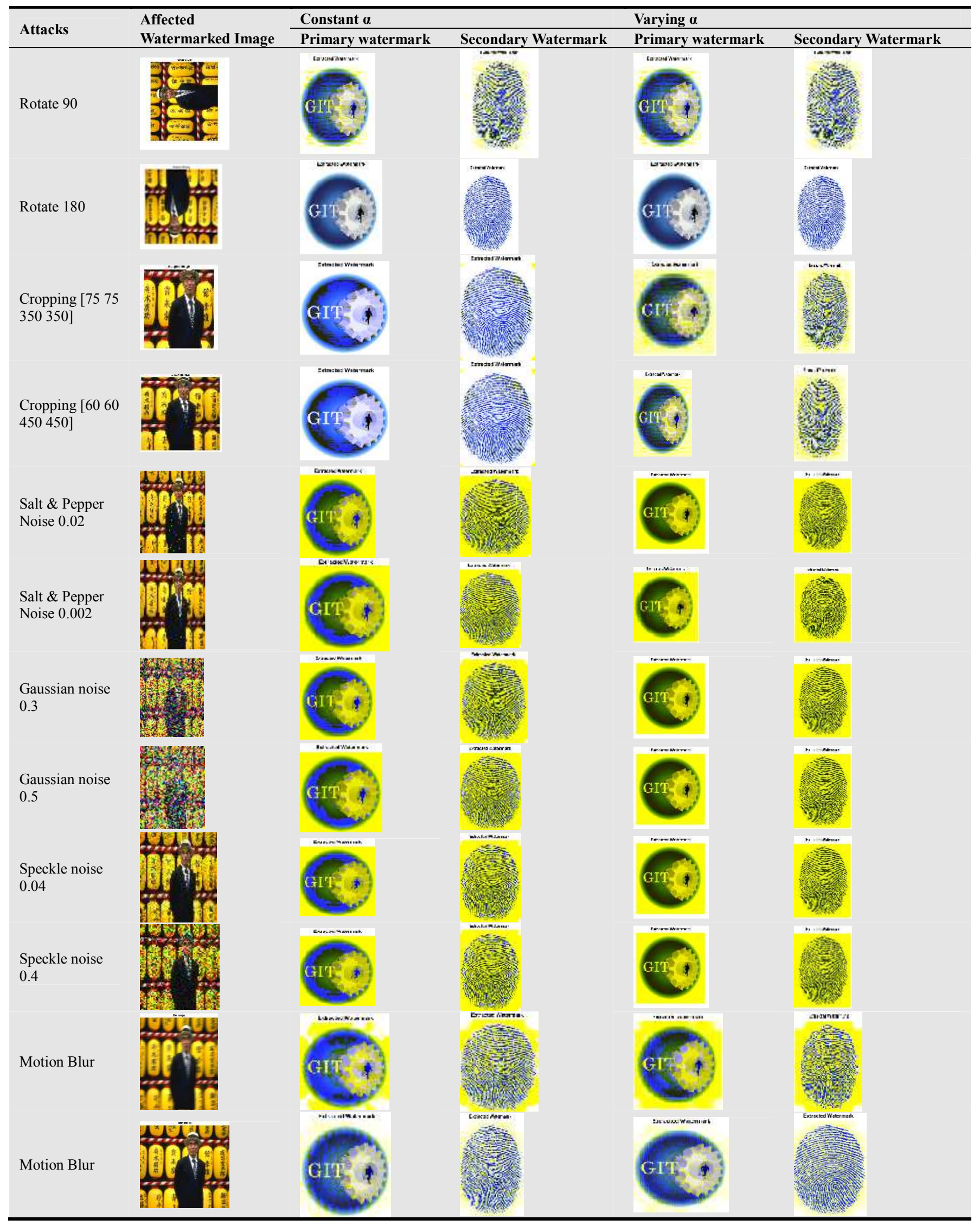




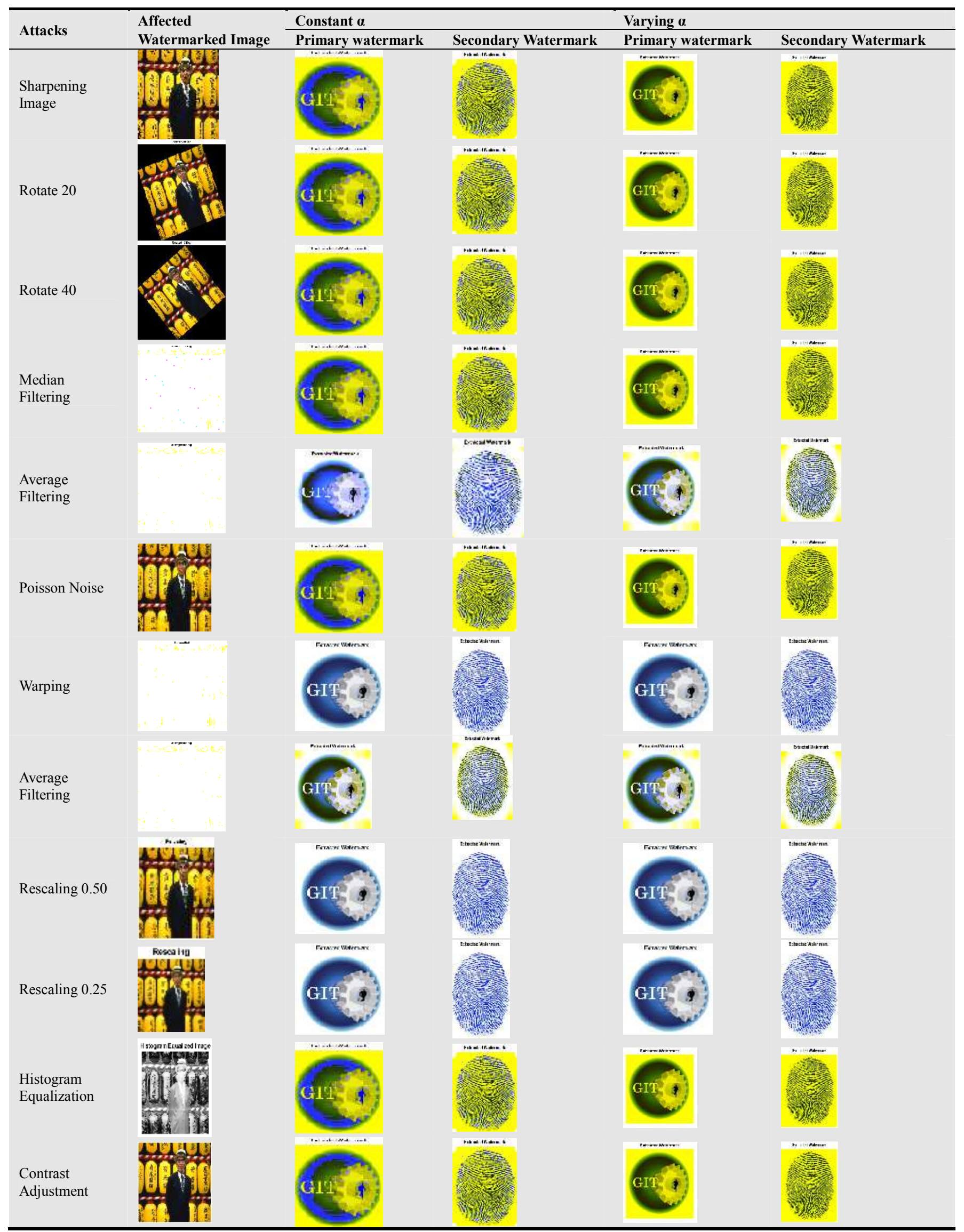




\begin{tabular}{|c|c|c|c|c|c|}
\hline \multirow{2}{*}{ Attacks } & \multirow{2}{*}{$\begin{array}{l}\text { Affected } \\
\text { Watermarked Image }\end{array}$} & \multicolumn{2}{|l|}{ Constant $\alpha$} & \multicolumn{2}{|l|}{ Varying $\alpha$} \\
\hline & & Primary watermark & Secondary Watermark & Primary watermark & Secondary Watermark \\
\hline $\begin{array}{l}\text { JPEG } \\
\text { Compression } \\
70 \%\end{array}$ & 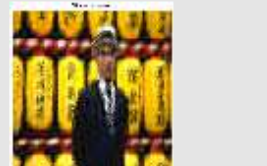 & & $\frac{4 x}{x y}$ & & 柆 \\
\hline $\begin{array}{l}\text { JPEG } \\
\text { Compression } \\
50 \%\end{array}$ & & & א. & & 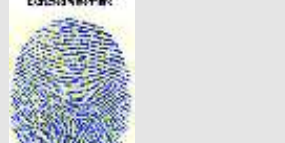 \\
\hline $\begin{array}{l}\text { JPEG } \\
\text { Compression } \\
30 \%\end{array}$ & 月 & & Whan & & Why \\
\hline $\begin{array}{l}\text { JPEG } \\
\text { Compression }\end{array}$ & ? & & & & W \\
\hline
\end{tabular}

Table 3. NC values of extracted watermarks with constant $\alpha$ and varying $\alpha$.

\begin{tabular}{|c|c|c|c|c|}
\hline \multirow{2}{*}{ Attacks } & \multicolumn{2}{|l|}{ Constant $\alpha$} & \multicolumn{2}{|l|}{ Varying $\alpha$} \\
\hline & PrimaryNC & SecondaryNC & PrimaryNC & SecondaryNC \\
\hline Rotate 90 & 0.7077 & 0.7480 & 0.7077 & 0.7480 \\
\hline Rotate 180 & 1 & 1 & 1 & 1 \\
\hline Cropping [ $\left.\begin{array}{lllll}75 & 75 & 350 & 350\end{array}\right]$ & 0.7284 & 0.7296 & 0.7284 & 0.7296 \\
\hline Cropping [ $\left.60 \begin{array}{llll}60 & 450 & 450\end{array}\right]$ & 0.8802 & 0.8948 & 0.8802 & 0.8948 \\
\hline Salt \& Pepper Noise 0.02 & -0.4718 & -0.4237 & 0.0688 & 0.1633 \\
\hline Salt \& Pepper Noise 0.002 & -0.4717 & -0.4237 & 0.0689 & 0.1635 \\
\hline Gaussian noise 0.3 & -0.4730 & -0.4252 & 0.0681 & 0.1626 \\
\hline Gaussian noise 0.5 & -0.4733 & -0.4256 & 0.0680 & 0.1624 \\
\hline Speckle noise 0.04 & -0.4722 & -0.4243 & 0.0685 & 0.1630 \\
\hline Speckle noise 0.4 & -0.4719 & -0.4238 & 0.0687 & 0.1632 \\
\hline Motion Blur & 0.3638 & 0.4481 & 0.3862 & 0.4862 \\
\hline Motion Blur & 0.9194 & 0.9362 & 0.9354 & 0.9516 \\
\hline Sharpening Image & -0.4717 & -0.4237 & 0.0691 & 0.1655 \\
\hline Rotate 20 & -0.2869 & -0.2295 & 0.1625 & 0.2583 \\
\hline Rotate 40 & -0.3646 & -0.3104 & 0.1521 & 0.2476 \\
\hline Median Filtering & -0.4717 & -0.4236 & 0.0698 & 0.1635 \\
\hline Average Filtering & 0.8484 & 0.8542 & 0.9386 & 0.9494 \\
\hline Poisson Noise & -0.4718 & -0.4237 & 0.0695 & 0.1645 \\
\hline Warping & 1 & 0.7340 & 1 & 0.9994 \\
\hline Average Filtering & 0.8484 & 0.8542 & 0.9395 & 0.9494 \\
\hline Rescaling 0.50 & 1 & 1 & 1 & 1 \\
\hline Rescaling 0.25 & 1 & 1 & 1 & 1 \\
\hline Histogram Equalization & -0.4750 & -0.4278 & 0.0675 & 0.1618 \\
\hline Contrast Adjustment & -0.4690 & -0.4206 & 0.0761 & 0.1708 \\
\hline JPEG Compression 70\% & 0.7090 & 0.7588 & 0.7090 & 0.7588 \\
\hline JPEG Compression $50 \%$ & 0.9448 & 0.9560 & 0.9448 & 0.9560 \\
\hline JPEG Compression $30 \%$ & 0.9924 & 0.9940 & 0.9924 & 0.9940 \\
\hline JPEG Compression $10 \%$ & 0.9998 & 0.9998 & 0.9998 & 0.9998 \\
\hline
\end{tabular}

In all those attacks the watermarked image is corrupted brutally, even though the watermarks are extracted back with good quality. Certain attacks like noising, filtering and histogram equalization the primary as well as secondary watermarks are terribly affected. By varying the value of $\alpha$ randomly between 0 and 1 both the watermarks are retrieved back with good quality. Table 2 shows that the normalized correlation values of primary and secondary watermarks using constant $\alpha$ and varying $\alpha$. Fig. 7 and 8 shows the normalized correlation values of primary and secondary watermarks have number of common similarity between different types of possible watermark attacks. Fig. 9 and 10 shows the primary as well secondary normalized correlation values in most common image processing attacks like removal and geometric attacks.

The Table2 shows the NC values are best maintained in 
attacks like Rotation, Motion blur, Average filtering, Warping, Rescaling and JPEG Compression and good in attacks like Blurring and Cropping and to be improved in attacks like Noising, Sharpening, Histogram Equalization and
Contrast adjustment. While comparing with existing techniques most of the normalized correlation values are improved except very few.

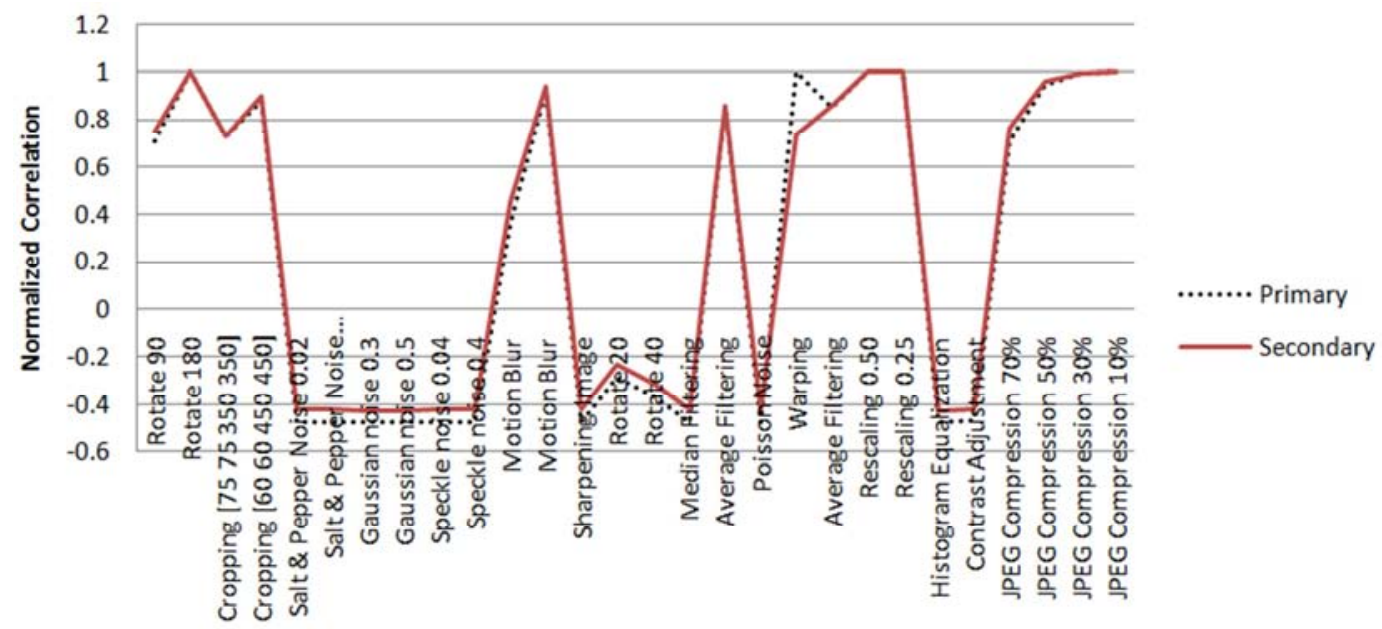

Fig. 6. NC values of extractedwatermarks with constant $\alpha$.

\section{Primary \& Secondary Watermark - Varying $\alpha$}

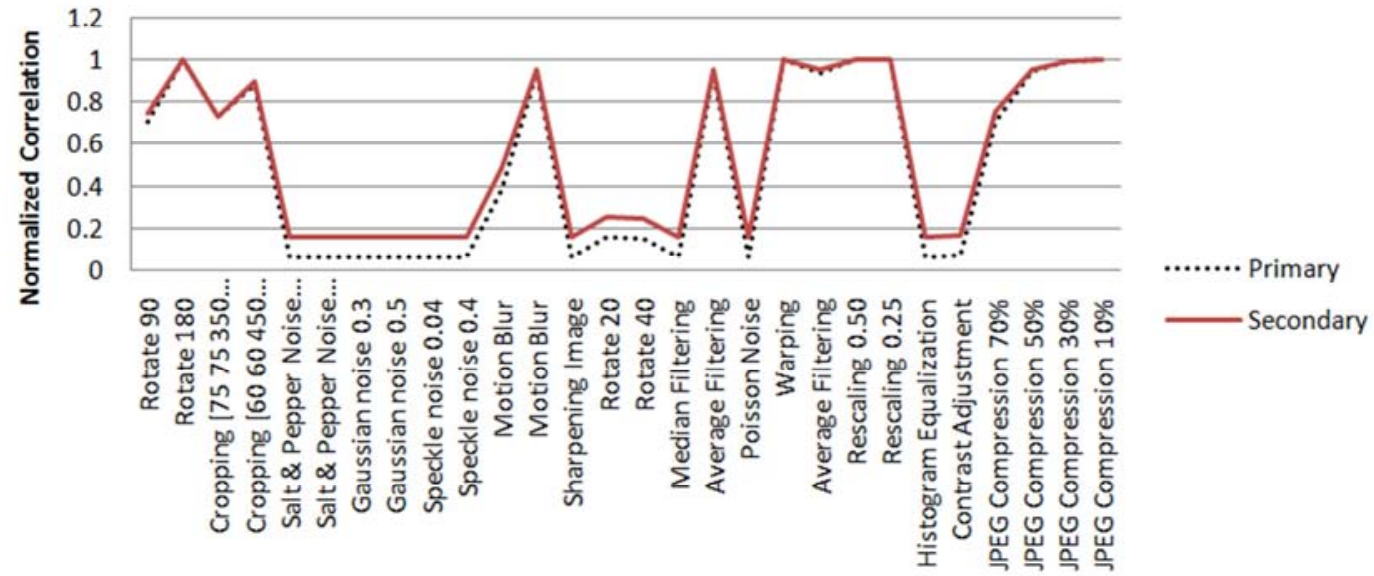

Fig. 7. NC values of extractedwatermarks with varying $\alpha$.

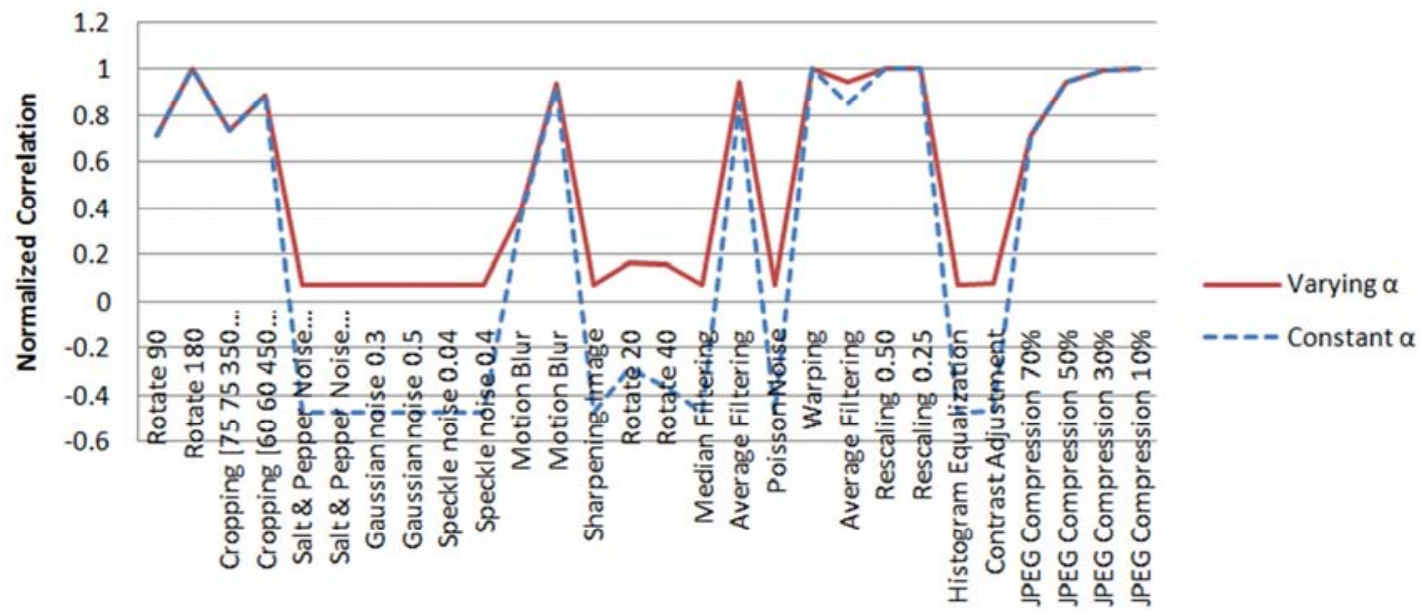

Fig. 8. NC values of extracted primary watermarks with constant $\alpha$ and varying $\alpha$. 


\section{Secondary Watermark}

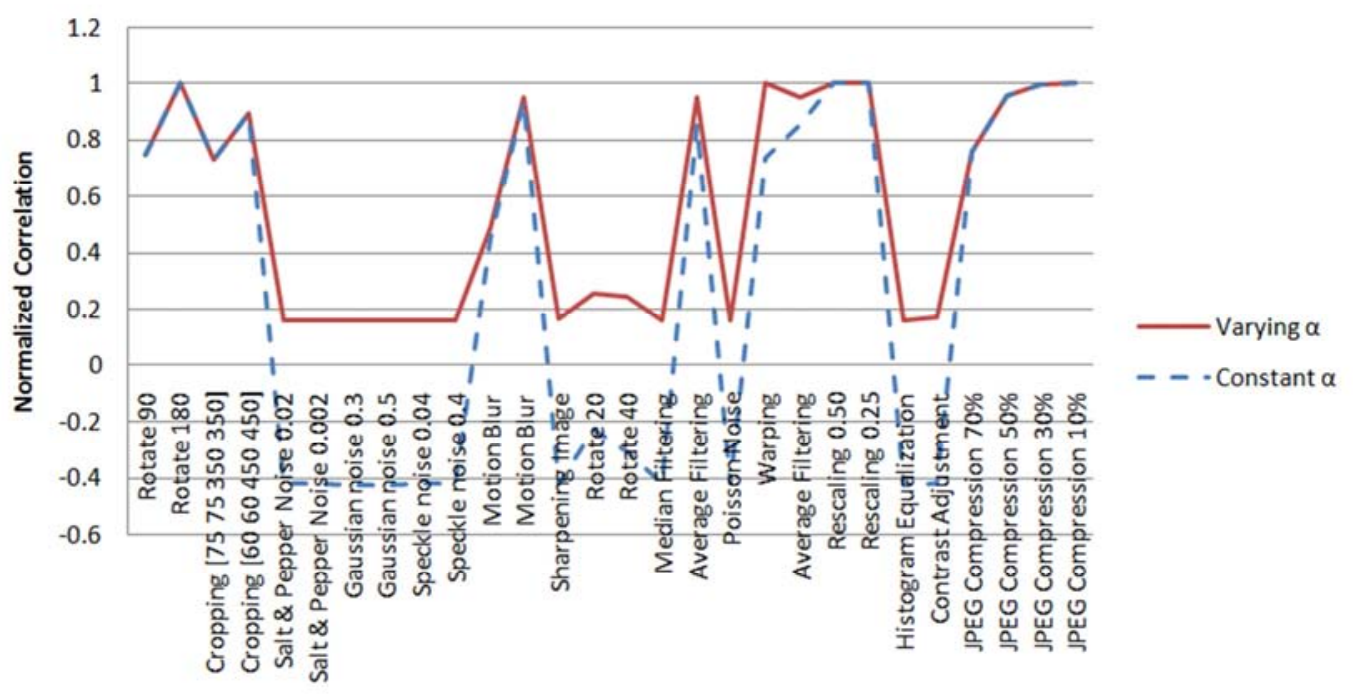

Fig. 9. NC values of extracted secondary watermarks with constant $\alpha$ and varying $\alpha$.

\section{Conclusion and Future Work}

The analysis was carried out by using an improved DWTDCT-SVD algorithm to fulfil the design criteria set to prove the hypothesis. The existing techniques have different sensitivities and robustness levels of different attacks of watermarks, but this algorithm shows the similarity between robustness levels of the extracted watermark. This proposed watermarking scheme not only guarantees the imperceptibility but also provides strong robustness to removal and geometric attacks. Both primary and secondary watermarks are extracted from the distorted watermarked image with high normalized correlation values after attempting most of the attacks. This algorithm reveals better performance in terms of image quality and robustness compared with the other related watermarking algorithm. The contribution of this paper to the advancement of digital image watermarking technology has summarised above. In future, an efficient algorithm may be developed to choose the random $\alpha$ value to adapt in the dual watermarking and the same may used in real world copyright protection systems.

\section{References}

[1] B. Subramanyan, Vivek.M. Chhabria, T. G. Sankar babu, "Image Encryption Based on AES key Expansion", IEEE Second International Conference on Emerging Applications of Information Technology, 2011, pp. 217-220.

[2] Vidyasagar M. Potdar, Song Han and Elizabeth Chang, "A Survey of Digital Image Watermarking Techniques", IEEE International Conference on Industrial Informatics, 2005, pp. 709-716.

[3] Cahndan Singh, Sukhjeet K. Ranade, "Image adaptive and high-capacity watermarking system using accurate Zernike moments", IET image process., 2014, Vol. 8, Iss. 7, pp. 373382 .
[4] Musrrat Ali and Chang Wook Ahn, Millie Pant, "An Optimized watermarking Technique Based on DE in DWTSVD Domain", IEEE, 2013, pp. 99-104.

[5] J. C. Liu, S. Y. Chen, "Fast two-layer image watermarking without referring to the original image and watermark", Image and Vision Computing 19(14), 2001, pp. 1083-1097.

[6] Taha Jassim and Read Abd-Alhameed, Hussain Al-Ahmad, “A New Robust and Fragile Watermarking Scheme for Images Captured by Mobile Phone Cameras", IEEE, 2013.

[7] Cox, IJ, Miller, ML \& Bloom, JA 2002, Digital Watermarking, Morgan Kaufmann Publisher, San Francisco, CA, USA.

[8] L. Ying - Hua, Q. Jing, K. Jun, L. Si - Hui, “A robust digital watermarking scheme based on optimal coefficients selector about sub images, wavelet analysis and pattern recognition", 2007, ICWAPR' 07, international conference, pp. 1865-1869.

[9] Kundur.D, Hatzinakos.D, "Digital Watermarking using Multiresolution Wavelet Decomposition", Proceedings IEEE International Conference on Acoustics, Speech and Signal Processing, Seattle, Washington, Vol.5,May 1998, pp. 29692972.

[10] Vinita Gupta, Mr. Atul Barve, “A Review on Image Watermarking and its Techniques", IJARCSSE, 2014, pp. 9297.

[11] A. Sverdlov, S. Dexter, and A.M. Eskicioglu, "Secure DCTSVD Domain Image Watermarking: Embedding data in all frequencies", Dept. Computer and Information Science, Brooklyn College, City Univ. of New York;http://brocklyncuny. academia.edu/ScottDexter/Papewrs/265184.

[12] Lai C C, Tsai C C, "Digital image watermarking using discrete wavelet transform and singular value decomposition", IEEE Transaction Instrumentation and Measurement 59(11), 2010, pp. 4707-4719.

[13] Zheng Xiong-bo, Zhang Xiao-wei and Sun Ming-jian, "A bling digital watermarking algorithm based on wavelet transform”, IEEE, 2011, pp. 679-682. 
[14] R. Liu and T. Tan, "An SVD-Based Watermarking Scheme for Protecting Rightful Ownership", IEEE Transactions on Multimedia, vol. 4, no. 1, 2002, pp. 121-128.

[15] Charles Way Hun Fung, Walter Godoy, “A Novel DWT-SVD video watermarking Scheme using side view”, IEEE, 2011.

[16] Cheng-dun Yin, Li Li, An-qiang Lv and Li Qu, "Color Image watermarking Algorithm Based on DWT-SVD", Proceedings of the IEEE International Conference on Automation and Logistics, 2007, pp. 2607-2611.

[17] Barin M, Bartolini F, Piva A, "Multichannel watermarking of color images", IEEE Transaction on Circuits and systems of Video Technology, 2002, vol. 12, no. 3, pp 142-156.

[18] Gaurav Bhatnagar, Q. M. Jonathan Wu, Balasubramanian Raman, "A new robust adjustable logo watermarking scheme" Computer Security $3^{\mathrm{I}}, 2012$, pp. 40-58.

[19] Podilchuk C I, Zeng W, "Image adaptive watermarking using visual models, IEEE J Select Areas Commun, 1998, pp.525539.

[20] Ganic E, Eskicioglu A. M, "Robust DWT-SVD domain image watermarking: embedding data in all frequencies", Proc. of the ACM Multimedia and Security workshop, 2004, pp. 166174.

[21] Song C, Sudirman S, Merabti M, "A robust region-adaptive dual image watermarking technique", J. Vis. Commun. Image R. 23, 2012, pp. 549-568.

[22] Makbol N. M, Khoo B. E, "Robust blind image watermarking scheme based on Redundant Discrete Wavelet Transform and Singular Value Decomposition", Int. J. Electron. Commun. (AEU)., 2012, http://dx.doi.org/10.1016/j.aeue.2012.06.008.
[23] Rastegar S, Namazi F, Yaghmaie K, Aliabadian A, "Hybrid watermarking algorithm based on Singular Value Decomposition and Radon transform", Int. J. Eletron. Commun. (AEU) 65, 2011, pp. 658-663.

[24] Chunlin Song, Sud Sudirman, Madjid Merabti, "A robust region-adaptive dual image watermarking technique", J. Vis. Commun. Image R. 23, 2012, pp. 549-568.

[25] Musrrat Ali, Chang Wook Ahn, Patrick Siarry, "Differential evolution algorithm for the selection of optimal scaling factor in image watermarking", Engg. Application of Artificial Intellignce. 31, 2014, pp. 15-26.

[26] Didi Rosiyadi and Shi-Jinn Horng, Pingzhi Fan, Xian Wang, Muhammad Khurram Khan and Pan Yi, "Copyright Protection for E-Government Document Images", Published by the IEEE Computer Society, 2012 IEEE.

[27] Niu Xiamu, Lu Zheming, Sun Shenghe, "Digital watermarking of still images with gray-level digital watermark", IEEE Transaction on Consumer Electronics, 2000, pp.1995-1998.

[28] Huang Jianyong, Yang Changsheng, "Image Digital Watermarking Algorithm Using Multiresolution Wavelet Transform", IEEE International Conference on System Man and Cybernetics, 2004, pp.2977-2982.

[29] A. Sverdlov, S. Dexter, A. M. Eskicioglu, "Robust DCT-SVD domain image watermarking for copyright pretection: embedding data in all frequencies", EUSIPCO2005, Antalya, Turkey, Sep-2005. 